\title{
The wind and wave atlas of the Mediterranean Sea - the calibration phase
}

\author{
L. Cavaleri \\ Institutte of Marine Sciences, ISMAR-CNR, S.Polo 1364, 30125 Venice, Italy \\ Received: 15 November 2004 - Revised: 16 July 2005 - Accepted: 18 July 2005 - Published: 21 July 2005
}

\begin{abstract}
Within the WW-Medatlas project, sponsored by the Italian, French and Greek Navies, an extensive atlas of the wind and wave conditions in the Mediterranean Sea has been completed. The atlas is based on the information derived from the archive of the European Centre for MediumRange Weather Forecasts, UK, then calibrated on the base of the data available from the ERS1-2 and Topex satellites. The calibration is required because the wind, hence the wave, data are normally strongly underestimated in the enclosed seas. The calibration has been done deriving the model values at each satellite position, typically at $7 \mathrm{~km}$ intervals. The colocated values have then been assigned to the closest grid point. This has provided a substantial number of couples of data at each point, then used to derive, by best-fitting technique, the correction required. This turns out to vary amply throughout the basin, according to the local geometry and orography. The calibration coefficients, different for wind and waves, have been used to correct the original fields and the time series at the single points. Using the calibrated data, extensive statistics have been derived, both as fields and at each point, including extreme values.
\end{abstract}

\section{Introduction}

The present state of the art of wind and wave modelling in the open oceans is pretty good. As an example, the comparison between the analysis fields of the European Centre for Medium-Range Weather Forecasts (ECMWF, Reading, UK) and the corresponding satellite data suggests a wind speed bias of the order of $10-20 \mathrm{~cm} / \mathrm{s}$ and a wave height bias close to $10 \mathrm{~cm}$. Simmons and Hollingsworth (2002) provide a good summary of the situation.

The overall conditions are less favourable in the inner seas, the Mediterranean being a classical example. Its dimensions are large, spanning more than $3600 \mathrm{~km}$ in longitude and more than 1600 in latitude. However, the complicated coastline, the protruding peninsulas, and the various large islands split the sea into a number of sub-basins of smaller dimensions. Here we find that the ECMWF wind speeds are substantially underestimated. Cavaleri and Bertotti (2004) have done a keen analysis of the situation, showing that the problem is connected to the proximity of land. It is found that the error is maximum where the wind blows off the land, with a trend to decreasing with fetch (length of sea run by wind), approaching the correct values, as measured by satellite, after several hundreds kilometres. In an enclosed sea practically all the marine areas are in this situation, and the model values turn out to be underestimated. The wave heights, strongly connected to the driving winds, are underestimated as well.

The problem can be alleviated using limited area, high resolution meteorological models (LAM). However, this solution is heavy in computer time and resources and cannot be applied systematically to long periods of time. This is critical, because, if we want to characterise a certain basin, we need at least ten years of data, and there is at present no way we can afford to run a LAM for such a period.

This paper deals with a solution to the above dilemma, that has made possible to obtain reliable long term time series of wind and wave parameters in the Mediterranean Sea, then used to derive spatial and point statistics throughout the basin. We believe these data are the best ones presently available in this basin.

In the next section we give a brief description of the climatology of the Mediterranean Sea, followed by the list of the data available and by a description of how they have been used to reach the final target. The last section provides some examples of the atlas tables. 
Topex altimeter data

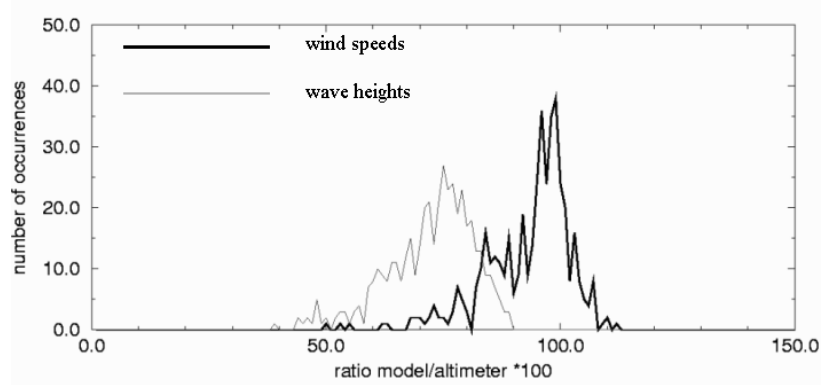

Fig. 1. Statistical distribution of the percent underestimate of the modelled wind speeds and wave heights in the Mediterranean Sea.

\section{The climatology of the Mediterranean Sea}

Enclosed between the storm belt of northern Europe and the tropical area of northern Africa, the Mediterranean Sea has a relatively mild climate on the average, but substantial storms are possible, usually in the six month winter. Cavaleri et al. (1991) provide a clear description of the situation. The maximum measured significant wave height reach $10 \mathrm{~m}$, but model estimates for some non-documented storms suggest much larger values. Even in the relatively small Adriatic Sea the oceanographic tower of ISMAR (Cavaleri, 2000), located on $16 \mathrm{~m}$ of depth, suffered heavy damage till $9 \mathrm{~m}$ above the mean sea level.

It is therefore relevant to obtain extensive reliable model data and the associated statistics. As explained in the next section, we need to make maximum use of all the available data.

\section{The calibration of the model data}

Neglecting the historical visual observations (their limitations have been clearly shown by Graham, 1982, 1983), we have available three sources of wind and wave data on the sea:

1. buoys and platforms - they provide continuous local time series, but they are relatively few and, especially buoys, mostly located close to the coasts,

2. satellites, with altimeters and scatterometers - they provide continuously data on the sea, but at continuously changing positions. At any specific point the altimeter data, the only one providing information on wave heights, are relatively few,

3. numerical models - continuous in space and time, typically at $25 \mathrm{~km}$ and $6 \mathrm{~h}$ intervals. However, as model data they are approximation to the truth, and mostly underestimated in the enclosed, hence Mediterranean, seas.

What we can do is to make a synergy of the overall data, taking each of them for which they are worth considering, and complementing each other deficiencies. The buoy data are used to cross-validate the satellite data. This was necessary because the calibration of the satellite instruments had been done mainly on the oceans. In principle, the different wave regime in the enclosed seas, characterised by a substantial lack of swell, could imply a different calibration. At least within the accuracy of our research, this was found not to be the case.

For our purposes we used the altimeter data of ERS1-2 and Topex. We could not make use of the scatterometer data because these had already been assimilated into the ECMWF model.

Then we proceeded to compare satellite and model data. First the Mediterranean Sea was divided in small areas of $0.5 \times 0.5$ degrees, centred on points distributed at 0.5 degree intervals throughout the basin. For each point we had available ten years of data, from July 1992 till June 2002, for wind and waves, at six hour intervals. For each satellite datum, for wind and wave separately, we have evaluated with a double interpolation in space and time the corresponding model datum. Then the co-located pair has been assigned to the closest point. This has made available at each point, at 0.5 degree intervals, a large number of pairs, of the order of thousands, whose scatter distribution has been best-fitted with a straight line. This has provided for each grid point, and separately for wind speed and wave height, the local model underestimate with respect to the satellite measured data.

Figure 1 provides a statistics of the underestimates throughout the grid. We see that, while a good percentage of the points has model/satellite wind ratios close to $1(100$ in the figure), hence with essentially no bias, at the majority of points the model data are underestimated, with minimum values close to 0.5 (50 in the figures). The situation is much worse for wave heights, with all the values well below the unitary value, down to almost 0.4 .

The inverse of these ratios are the calibration factors by which the model data have to be multiplied. This has provided ten years of calibrated wind and wave data throughout the basin, suitable for a statistical analysis.

We have also done an estimate of the accuracy of the calibration. First, we validated the satellite data versus buoy data. In practice this was possible only for wave height, as wind data, as measured from buoys and marine platforms, are extremely scarce in the Mediterranean Sea. Then we considered the scatter of the single distributions between altimeter and buoy data.. The critical areas turned out to be the most enclosed seas (Ligurian, Adriatic, Aegean) and in general the ones close to the coasts, in connection with the related difficulties of the satellite measurements. This has suggested to smooth the overall distribution of the calibration factors.

Beside overall distributions, the atlas provides statistics at about 200 points, distributed at one degree intervals, denser in the mentioned more critical areas. 


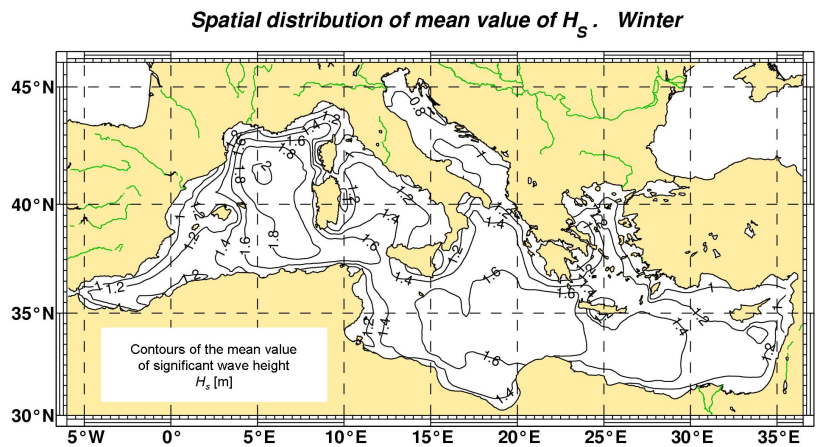

Fig. 2. Distribution of the winter mean significant wave height in the Mediterranean Sea. The isolines are at $0.2 \mathrm{~m}$ intervals (after The Medatlas Group, 2004).

\section{Statistics}

Figure 2 shows the distribution of the mean significant wave height throughout the basin. The isolines are traced at $0.2 \mathrm{~m}$ intervals. The area with the strongest average activity is the gulf of Lion, in the Western Mediterranean Sea. The statistics are provided both on annual and seasonal bases.

The available statistics concern wind speed and direction, and wave height, period and direction. For each of the about 200 points there are related, both uni- and bi-modal, distributions, available as diagrams and ASCII tables.

The atlas is available from the author both in paper and electronic format. The front page of the atlas is shown in Fig. 3.

Acknowledgements. The original model data have been obtained from the archive of the European Centre for Medium-Range Weather Forecasts (Reading, UK).

The project has been a common effort between ISMAR-CNR and Thetis (Italy), Meteo-France, CS-SI and Semantic (France) and NTUA (Greece). It has been sponsored by the Italian, French and Greek Navies, via WEAO project RTP 10.10.

Edited by: L. Ferraris

Reviewed by: R. Crapolicchio and another referee

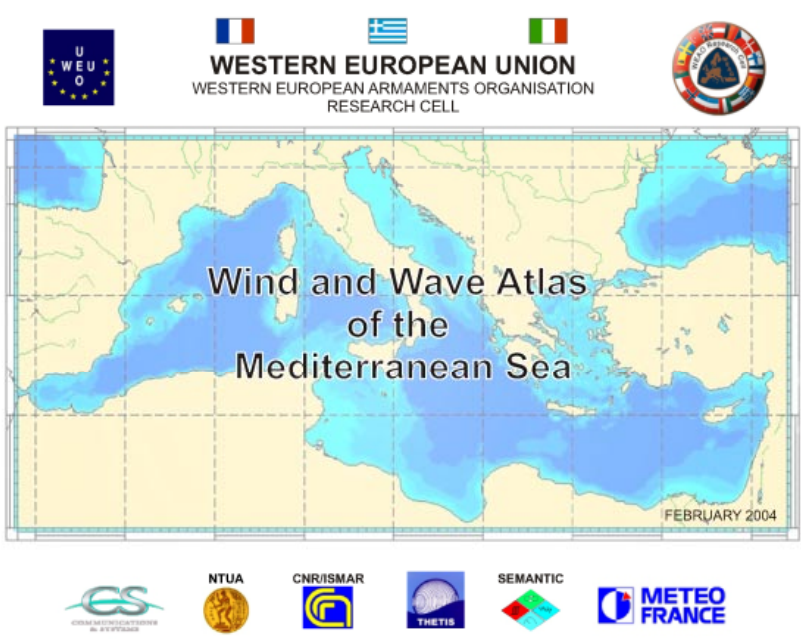

Fig. 3. The cover of the WW-Medatlas atlas.

\section{References}

Cavaleri, L. and L.Bertotti: Accuracy of the modelled wind and waves in enclosed seas, Tellus, 56A, 167-175, 2004.

Cavaleri, L.: The oceanographic tower Acqua Alta - activity and prediction of sea states at Venice, Coastal Engineering, 39, 2970, 2000.

Cavaleri, L., Bertotti, L., and Lionello, P.: Wind wave cast in the Mediterranean Sea, J. Geophys. Res., 98, C8, 10739-10 764, 1991.

Graham, A. E.: Winds estimated by the voluntary observing fleet compared with instrumental measurements at fixed positions, Meteorological Magazine, 111, 312-327, 1982.

Graham, A. E.: Wave heights estimated by the voluntary observing fleet compared with instrumental measurements at fixed positions, Meteorological Magazine, 112, 210-224, 1982.

Simmons, A. J. and Hollingsworth, A.: Some aspects of the improvement in skill of numerical weather prediction, Q. J. Roy. Meteor. Soc., 128, 647-677, 2002.

The Medatlas Group: Gaillard, P., Ravazzola, P., Kontolios, Ch., Arrivet, L., Athanassoulis, G. A., Stefanakos, Ch. N., Gerostathis, Th. P., Cavaleri, L., Bertotti, L., Sclavo, M., Ramieri, E., Dentone, L., Noel, C., Viala, C., and Lefevre, J.M.: Wind and Wave Atlas of the Mediterranean Sea, 420pp. 